\title{
Reconstruction of Cellular Biological Structures from Optical Microscopy Data
}

\author{
Kishore Mosaliganti, Lee Cooper, Richard Sharp, Raghu Machiraju, Gustavo Leone, Kun Huang and Joel Saltz
}

\begin{abstract}
Developments in optical microscopy imaging have generated large high-resolution datasets that have spurred medical researchers to conduct investigations into mechanisms of disease, including cancer, at cellular and sub-cellular levels. The work reported here demonstrates that a suitable methodology can be conceived which isolates modality- dependent effects from the larger segmentation task and that $3 D$ reconstructions can be cognizant of shapes as evident in the available $2 D$ planar images. In the current realization, a method based on active geodesic contours is first deployed to counter the ambiguity that exists in separating overlapping cells on the image plane. Later, another segmentation effort based on a variant of Voronoi tessellations improves the delineation of the cell boundaries using a Bayesian formulation. In the next stage, the cells are interpolated across the third dimension thereby mitigating the poor structural correlation that exists in that dimension. We deploy our methods on three separate datasets obtained from light, confocal and phase-contrast bright field microscopy and validate the results appropriately.
\end{abstract}

Index Terms - cellular reconstruction, segmentation, tessellations, microscopic imaging

\section{INTRODUCTION}

In this work, we focus on the three-dimensional reconstruction of microscopic cellular structures. A reconstruction of cellular structures when combined with genetic/molecular expressions will further the understanding of disease [2], [19]. More importantly, three-dimensional reconstruction will allow biologists to see beyond the $2 D$ image planes that they are accustomed to.

Figure 1(a) shows a typical image of a tissue employed in prototypical phenotyping studies [14]. Note the clear lack of a global $3 D$ description of microscopic structures that populate each of the image planes. The cells on each plane are best identified by the presence of nuclei; however, the extent and proliferation of the nuclei in typical cellular constellations is not easily demarcated in $3 D$. Further, tubular structures (e.g., ducts) which are prevalent in lesser numbers can only be identified on each slide as a projection of a complex tortuous 3D object. The imaging plane does not slice the 3D object normal to its axis and hence the profile on consecutive projections is prone to rapid changes in consecutive slices (Figure 7a). Transfer function based visualization fails to

K. Mosaliganti, R. Sharp and R. Machiraju are with the Departments of Computer Science and Engineering at The Ohio State University. email: \{mosaligk, sharpr, raghu\}@cse.ohio-state.edu

L. Cooper, K. Huang and J. Saltz are with the Department of Biomedical Informatics at The Ohio State University. email: $\{$ lee, khuang,saltz $\} @$ bmi.ohiostate.edu

G. Leone is with the Department of Human Cancer and Genetics at The Ohio State University. email: gustavo.leone@osumc.edu resolve hidden $3 D$ structures since the structural correlation across image planes is only partial (see Figure 1(c)).

The process of acquisition (slicing, staining etc) creates poor $3 D$ correlation in image color, gradients and structures. Even after corrective registration procedures, one realizes that the sections are not fine or thin enough to capture $3 D$ profiles of cells/nuclei. Reconstruction of microscopic structures, on the other hand, allows for the easy location and measurement of changes in the cellular complex as a result of biological events including phenotyping. The end result of such an effort is a set of segmented and labeled structures. Rendering these segmented and labeled structures allows for further visual interpretations of the data.

Figure 2 motivates the recurring problems with yet another microscopy dataset - confocal images of the zebra fish embryo. The dataset describes an evolving embryo from seed cells into a significant critical mass. Nevertheless, we observe that renderings of raw data cause the cells to appear diffused in space. In confocal microscopy, the sectioning is accomplished optically. However, the optical resolution along the $z$-axis is limited and results in poor resolution of structures across the principal $x y$ planes.

Proposed Approach: Our framework is composed of three conceptual stages. The first stage is modality-dependent, wherein a coarse segmentation result is obtained. This stage uses a shape model of a nucleus. The second stage is less dependent on the modality and improves the results of segmentation by exploiting knowledge of cellular arrangements. The third component allows the extrapolation of shapes into the third dimension $(z-$ axis $)$. This separation of the reconstruction process into these three stages permits the development of robust and flexible algorithms. Thus, a single methodology and a comprehensive software suite can effectively process and analyze microscopy data from a variety of sources and our approach marks a departure from normal practice, whereby tools are developed separately for each modality.

i. Modality-dependent Segmentation: Given the variability in microscopy data, segmentation is usually the first step of any analysis protocol. The nucleus/cell forms the fundamental biological entity of interest. Often, nuclei appear as overlapping or touching each other on $2 D$ image planes. An image of a nucleus is formed from an orthographic projection of the $3 D$ structure in a slice when light traverses through it. Hence, in reality, it is possible that the nuclei do not even touch each other in $3 D$. Identifying each nucleus separately in a biologically consistent fashion is non-trivial. While some histological stains provide viable clues in the form of sharp color-space gradients at the boundaries [4], others exhibit a 


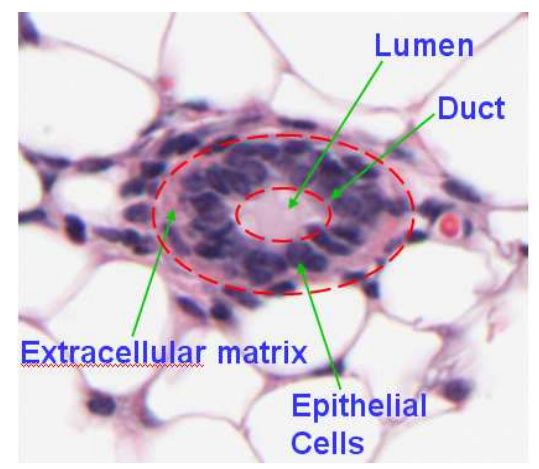

(a)

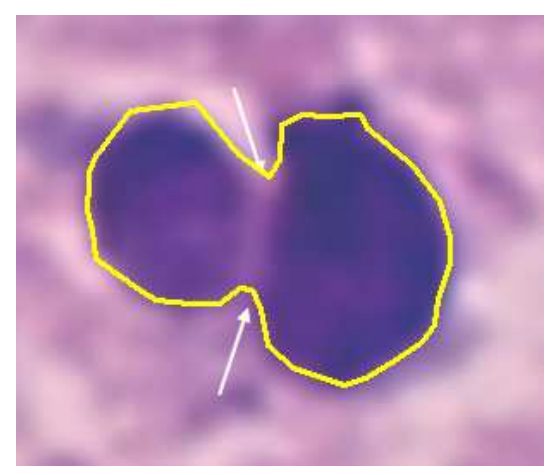

(b)

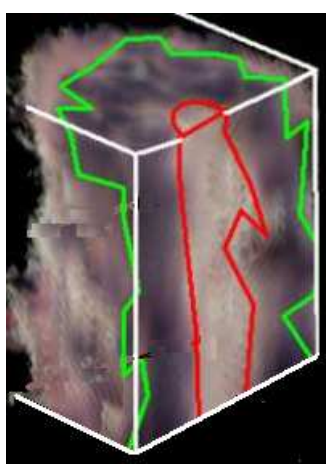

(c)

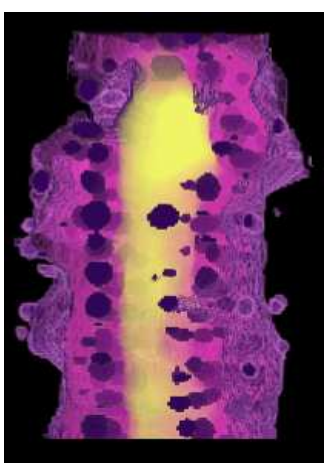

(d)

Fig. 1. Reconstruction challenges of histological serial sections from light microscopy. (a) A section of a mice mammary duct with labeling. (b) Zoomed versions showing two nuclei that appear coalesced together. The yellow contour represents the nucleus foreground. Note the presence of a neck region (white arrows) marked by color intensity gradients. (c) Visualization of a duct axial cross-section reveals no cellular information. (d) Enhanced visualization showing ductal epithelial nuclei after applying our framework.

narrow neck at the site of overlap between two nuclei [25]. Please refer, for example, to Figure 1(b) wherein a pair of nuclei are shown with color gradients and a narrow neck indicated by the white arrows. Nevertheless, there is no approach that elegantly incorporates all available cues and shapes into a viable segmentation exercise. We implement a geodesic active contours solution incorporating shape priors in a level-set framework to provide coarse segmentations that can distinguish and mark neck regions. It should be noted that the initial segmentation for the active contours evolution can be achieved from the use of a modality-dependent threshold or a template (derived from the point-spread function of the instrument).

ii. Modality-independent Segmentation: The abovedescribed level-set framework provides tuneable parameters to account for modality-specific effects such as image intensities, gradients, and nucleus shapes in determining a coarse segmentation. However, there is tremendous variation of the nucleus entity as captured in digital form in the same data. There are several biological reasons as to why the color and shape of a given entity varies; the stage in the cell-division cycle, the effect of malignancy and other reasons. At best, the parameter settings of the earlier stage describe an average cell in the given ensemble.

One of the novel contributions of this work, is the use of tessellations to provide refined segmentations. Rather than trying to capture boundaries correctly, a more meaningful solution is to tessellate the image into regions that house an individual nucleus completely. This stage makes use of the coarse segmentations from the earlier stage. Our initial tessellation-of-choice is based on the centroidal Voronoi tessellations (CVT) [7]. The optimal placement of the tessellating lines are guided by a Bayesian probabilistic model of nuclei separation. There is yet another motivation for using the tessellation strategy.

Cells as opposed to nuclei are not visible under popular staining protocols such as those used in histology (H\&E) or confocal images (Draq5). In such circumstances, the following question arises: how does one place limits on the cell extents. Cell membranes have complex topology that is not yet completely understood. Hence, each demarcated region in a tessellated space neatly encapsulates a cell (containing the nucleus) ideally and elegantly delineates all natural neighbors (necessary for characterizing). Our philosophy of using such an approach originated after long interactions with the biologists and determining their needs.

iii. Intermediate Slice Interpolation: The correspondence of nucleus profiles is not maintained along the stacking direction. Assuming that a nucleus is roughly spherical in shape, the inter-slice thickness is much larger than the nucleus diameter. Under-sampling artifacts are visible during the 3D visualization of the stack. For instance, all the nuclei profiles are only visible as a dark ductal wall in Figure 1(c). Similarly, in Figure 2(b), nuclei appear to be linearly stretched. In our approach, intermediate slices are interpolated between any two existing slices based on a derived nucleus shape model and nuclei locations observed in the terminal image planes.

Thus, we obtain effective high-resolution reconstruction of the micro-cellular anatomy as shown in Figure 1(d) and Figure 2(d). Note the clearly demarcated ductal walls and cell boundaries, thus mitigating the two prominent ill-effects of serial-section imaging depicted in Figure 1(b), Figure 1(c) and Figure 2(b). Figure 8 and Figure 10 show additional renditions of the reconstructed cellular environments in light and confocal data respectively.

The rest of the paper is organized as follows. In Section II, we describe related work in visualization and imaging forums. Section III explains our $2 D$ cellular segmentation methods, while Section IV introduces our method of tessellations. Later Section $\mathrm{V}$ describes the generation of intermediate slices with a nucleus model. Section VI showcases some of our reconstruction and validation efforts when applied to three different microscopy modalities. Finally, in Section VII, we provide a summary and describe our plans for the future.

\section{RELATED WORK}

In this section, we will review work relevant to $3 D$ visualization and reconstruction of cellular data from microscopy. Where necessary, other relevant work pertaining to level-set 


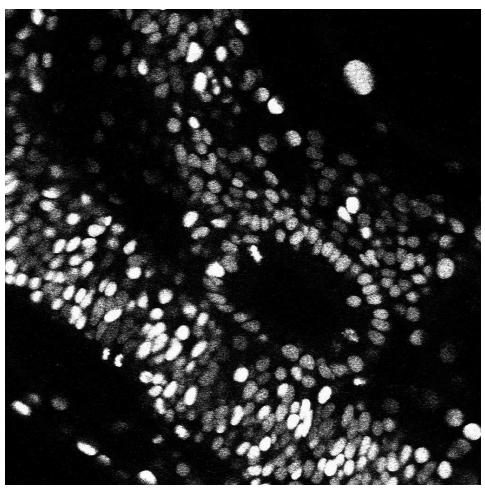

(a)

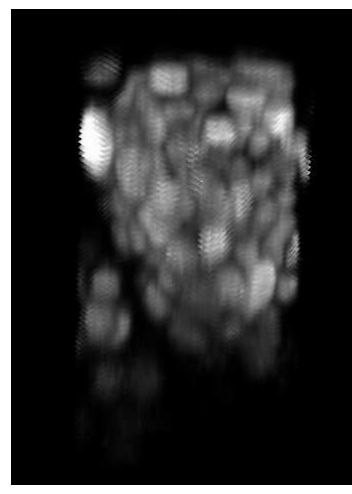

(b)

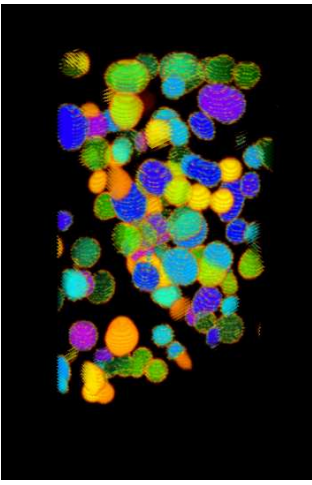

(c)

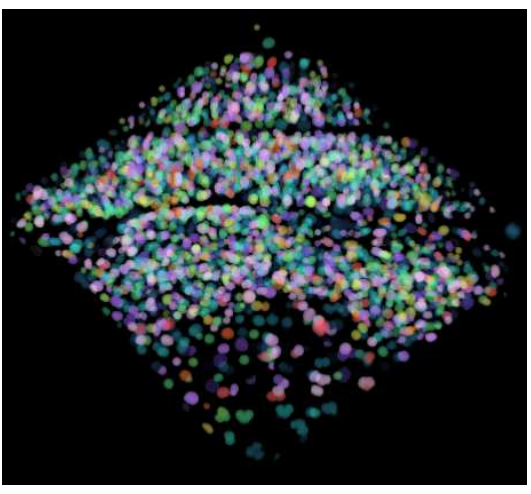

(d)

Fig. 2. Reconstruction challenges in confocal images. (a) A typical image showing biological structure composed of cells of the embryo of a zebra fish. (b) A $3 D$ rendering of a small volume in the original data with appropriate voxel spacing. (c) A reconstruction of the same volume using cell segmentations and interpolation. (d) A rendering of all the segmented cells.

techniques, general cell segmentation approaches and tessellations will be described.

Our goals require us to achieve reconstruction at a cellular level in the presence of serious under-sampling. A $3 D$ visualization/reconstruction algorithm may only be deployed when the dataset is registered, has sufficient sampling resolutions in all three dimensions and presents sufficient continuity in colors, gradients and illumination etc. Hence, direct volume rendering using transfer functions has limited scope of application. Early visualization research of confocal microscopy data takes cognizance of these difficulties [13]. Existing visualization research in this domain has been restricted to developing GPU-based techniques [27], hardware accelerated rendering software and algorithms [5], [22] and texture-based transfer functions [26].

In serial-section microscopy, information is available on a per image basis with little or no correspondence of structures at a cellular level. Typically, one segments each image into various components and regions and then achieves alignment across the images through a process of registration [3], [9]. Work presented in [8] follows this mantra successfully to achieve tangible reconstructions. We employ a similar strategy towards reconstructing $3 D$ cellular geometry. However, as mentioned earlier in Section I, we address specific challenges that these tasks pose for the given data.

Previously, in [23], we described a pipeline to visualize histological stacks derived from the mice placenta organ. The emphasis therein was not on reconstructing the cellular geometry. We use level set methods to obtain coarse $2 D$ cellular segmentation. The geodesic active contours approach with a level-set implementation causes the contour to halt at object boundaries exactly. Leventon et al. [15] extended the geodesic active contours method with an additional shapeinfluenced term in the driving PDE using a maximum $a$ posteriori (MAP) estimate of the position and shape of the object in the image.

We refine our coarse segmentations using tessellations embedded in the image. Tessellations divide a region using geometric hyperplanes thereby isolating the entities-of-interests (e.g., nuclei). The centroidal Voronoi tessellations (CVTs) have inspired the development of several useful segmentation and visualization algorithms [7]. Recently, Du et al. [7] showed the applications of CVT-based vector quantization to the visualization of flow vectors. In our case, the CVTs are not always the optimal tessellations in the presence of overlapping and anisotropic nuclei shapes. Mosaliganti et al. [17] developed a new representation of the CVTs for clustering applications. The CVTs were parameterized in a barycentric coordinate representation space of the Voronoi centers. This provided flexibility in re-defining a Voronoi cell for more meaningful tessellations.

The segmentation of overlapping nuclei in a biologically consistent manner is an active topic of research. There is a plethora of reported work that uses morphological operators and application/modality specific features. These approaches are not often generalizable. In [16], the image is treated as a height field and watershed segmentation is performed to determine cell boundaries. This approach is fragile because small islands of noisy pixels can cause over-segmentations. Tscherepanow et al. [25] presented an active contour approach with a snakes formulation wherein overlapping cells are segmented as dictated by a $2 D$ elliptical model. Jones et al. [4] presented a novel method that first defines a metric in the image plane for calculating distances from seed regions. Pixels are then assigned to cells according to their distance from the corresponding nucleus under that metric. The metric uses information about image edges, both their strength and their orientation, as well as a regularization term corresponding to inter-pixel distance within the image. In the ideal case, their method will create tessellations similar to ours. However, their chosen metric is not often very discriminating when the neck cues are weak.

All of the approaches discussed above rely on using information derived either from cellular shape models [25] or internuclear gradients [4] or neck shape cues [24]. It is interesting to note that no single approach elegantly incorporates all the cues into the segmentation process. In contrast, our active contour approach implements the three aspects into a compre- 


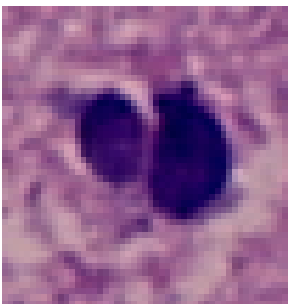

(a)

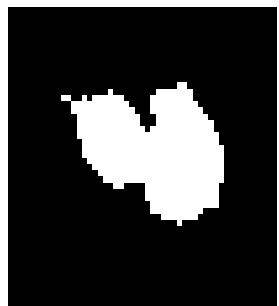

(b)

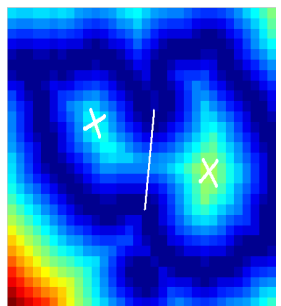

(c)

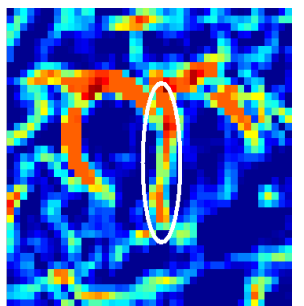

(d)

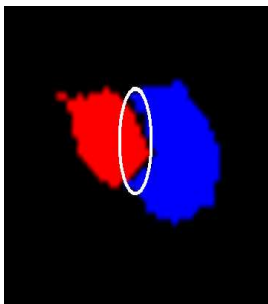

(e)

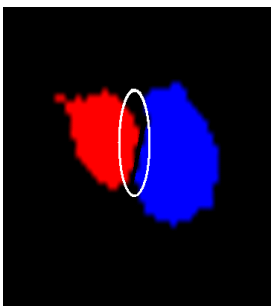

(f)

Fig. 3. Nuclei segmentation using geodesic active contours. (a) A pair of overlapping nuclei. (b) Applying a threshold $T=100$ on the red channel of the color image provides the nuclei foreground $I_{f}$ and background $I_{b}$. (c) Unsigned distance field $P$ of the nuclear contour. The nuclei centers ('x') are located at maxima and the neck region is in a directional minimum (white line segment). (d) High gradient magnitudes $|\nabla I|$ typically exist in the neck region although this is not guaranteed. (e) Segmentation obtained using geodesic active contours. (f) Improving the segmentation by using linear tessellations.

hensive framework through suitably defined speed functions in the level-set formulation. Additionally, the use of tessellations will increase the robustness of our approach. We now describe our methods in subsequent sections.

\section{Modality-dependent Segmentation: Active CONTOURS MODELS}

Three-dimensional segmentation algorithms will incorrectly delineate complete cells given the problems of under-sampling that exist in the image data. We use the active contour models with a level-set implementation for obtaining an initial $2 D$ segmentation of the nuclei. The two key steps for the level-set method are:

1) Embedding the surface: we represent a given nucleus contour $\Gamma(t)$ as the zero level-set of the signed distance function $\psi(\mathbf{x}, t)$. Formally, $\Gamma(t)=\{\mathbf{x}: \psi(\mathbf{x}, t)=0\}$.

2) Embedding the motion: we derive the update equation 1 such that the motion of the zero level set has certain desired properties described later. For this purpose, we use the active contour formulation with shape priors developed by Leventon et al.:

$$
\frac{\partial \psi}{\partial t}=f(I)(\alpha c+\beta \kappa)|\nabla \psi|+\gamma \nabla f . \nabla \psi+\delta \gamma\left(\psi^{*}-\psi\right)
$$

The function $f$ refers to the image-based feature function that is minimized at a nucleus boundary and remains high elsewhere. The function $f$ involves information drawn from the image gradients and neck artifacts. We will return to it later in the discussion.

The first speed term $(c+\kappa)|\nabla \psi|)$ consists of the curvaturedependent and propagation-dependent speed terms. The parameter $c$ is a balloon force that is added to evolve the curve outwards and $\kappa$ is the curvature along the normal to the levelset contour. Since the boundary is characterized by a local minimum in $f$, the term $\nabla f . \nabla \psi$ constitutes the boundary attraction term. This ensures that the contour lies on the nucleus boundary upon convergence. The final term $\left(\psi^{*}-\psi\right)$ is a recent addition by Leventon et al. [15] wherein they incorporated principal components of the segmentation shape model to drive the update equation. The surface $\psi^{*}$ is the maximum a-posteriori shape given the current segmentation and image information. The parameters $\alpha, \beta, \gamma$ and $\delta$ are user-defined settings for the relative scaling of the three speeds and the model-driven update.

We incorporate three ideas from literature that were developed independently namely, (i) shape models (already described in the third speed term), (ii) inter-nuclear boundary gradients and (iii) neck shape cues.

Shape Model: Training data consisting of manually segmented nuclei from $2 D$ images was used in estimating a PCA-based shape model with three modes of variation in a manner similar to that described in [15]. The advantage of using this model is that a good number of cells within a phenotype share similar sizes, shapes and even orientations. Hence this information is incorporated as the MAP shape $\psi^{*}$ in guiding the evolution in Equation 1.

Image feature function $f$ : Please refer to Figure 3 for an illustration of the associated concepts. Let $I$ represent a typical image with well-contrasted nuclei that needs to be segmented (Figure 3a). A threshold is applied to separate the nucleus foreground $\left(I_{f}\right)$ from the background $\left(I_{b}\right)$ (Figure $3 \mathrm{~b}$ ). Formally:

$$
I_{f}(x, y)= \begin{cases}0, & \text { if } I(x, y)>T \\ 1, & \text { if } I(x, y) \leq T\end{cases}
$$

Let $D\left(I_{f}\right)$ and $D\left(I_{b}\right)$ represent the unsigned distance fields emanating from the threshold contour that exist outside and within the contour respectively. Consider the image formed by $P=D\left(I_{f}\right)+D\left(I_{b}\right)$ shown in Figure 3(c). $D\left(I_{b}\right)$ causes the appearance of a directional minimum in the neck region as represented by a white line segment. The appearance of a directional minimum is a direct consequence of the neck shape resulting in lower magnitudes of the distance field. At the same time, the nucleus-background boundary is marked by a 0 isovalue in $P$ by definition, and positive elsewhere. Both these reasons contribute in halting the evolution that results in nuclei segmentation. While the presentation so far accounts for the neck cues, we also observe that some nuclei exhibit high gradients at the site of overlap as shown in Figure 3d. As in standard practice, we take the gradient information, $g(I)$ into account. Our feature function is therefore defined as:

$$
\begin{gathered}
f=\frac{g(I)+\eta\left(D\left(I_{f}\right)+D\left(I_{b}\right)\right)}{1+\eta} \\
g(I)=\frac{1}{1+\left|\nabla G_{\sigma} * I\right|^{2}}
\end{gathered}
$$


The gradient information $g(I)$ is the reciprocal function of the image gradient magnitudes. The image gradients are computed using a standard derivative-of-Gaussian (DoG) filter with an appropriate $\sigma$. The parameter $\eta$ represents a scaling factor ranging from $[0, \infty)$. For large values, the neck cues are assigned more weight than the inter-nuclear gradients $g(I)$. The level-set initialization occurs inside each nucleus. Hence we apply a threshold on image $P$ and choose the resulting iso-contour lying within the nucleus foreground as our initialization.

\section{Modality-Independent Segmentation: Using TESSELLations}

We observe that the level-set segmentation procedure described earlier does not produce consistent boundaries when separating nuclei clusters as shown in Figure 3e. This is because the feature function is defined using distance maps that are, in turn, sensitive to noisy contours. Furthermore, the standard level-set parameter settings do not effectively capture the variations in nuclear shape and inter-nuclear gradients. This leads to inconsistent segmentations especially at boundaries.

While it may be argued that this is a consequence of using the level-set methodology, related work in literature shows that this problem has been a recurring one as far as microscopic datasets are concerned. As we motivated earlier in Section I, the staining protocol, the slicing methods etc. take a heavy toll on the quality of the images generated. The variations are ubiquitous to microscopy datasets and prove to be an Achilles' heel for standard segmentation algorithms. For example, Adiga et al. [1] reported on an advanced watershed segmentation with a post-processing mechanism to correct the over-segmentations. A rule-based merging scheme based on heuristics was setup. Unfortunately, these heuristics are application-specific and lack a rigorous framework of implementation. In a similar vein, Raman et al. [20] reported on the use of morphological operators and connected components to obtain a coarse segmentation. A set of heuristics were applied that naturally led to an approach similar to one based upon tessellations. Their groupings required placing lines across novel contour points of positive curvature and enforcing constraints such as the non-intersection of separating line segments, convexity, and average area of the partitioned nuclei.

We now describe a rigorous, novel post-processing technique that seeks to correct the boundaries. We make use of the naturally occurring Voronoi tessellations to generate our line segments. These segments are altered using a Bayesian probabilistic framework. As a result, new heuristics that may occur in specific applications are naturally incorporated into the model. It marks a significant shift in philosophy - isolate image regions having a salient nucleus rather than extracting individual nucleus from a large image.

\section{A. Algorithm Overview}

The algorithmic intuition is best explained as a sequence of steps along with a running example drawn from the mammary duct dataset. Please refer to Figure 4. We deploy the geodesic active contours algorithm on the given data to obtain coarse nuclei segmentations (Figure 4(b)). To correct, we do as follows:

1. The Voronoi tessellation of the image (Figure 4(c)) is constructed using the centroid of the identified nuclei as the generator set. The nucleus that is housed in a Voronoi cell may infiltrate into neighboring Voronoi cells and vice-versa (Figure 4(d)). We now rectify the tessellation to correctly separate nuclei.

2. The set of Delaunay triangles dual to the Voronoi map is used in modulating the tessellations. We obtain the parameterized barycentric representation of the Voronoi tessellation line using a local Delaunay triangle as outlined in Section IV-C.

3. We determine the maximum a posteriori (MAP) line parameters given image information, preliminary segmentations and a set of heuristic rules. As a result, more viable tessellation lines are chosen for the final segmentation as shown in Figure 4(d). Now, the nuclei are deemed to be separated and used in the interpolation framework.

\section{B. Voronoi Tessellations - Step 1}

Given a set of generating points $V_{i} \in \mathfrak{R}^{2}$, the Voronoi map is a partitioning of $\Re^{2}$ using line segments into salient regions. Each region $Z_{i}$ corresponding to the generator $V_{i}$ is defined by:

$$
Z_{i}=\left\{V \in \Omega:\left|V-V_{i}\right|<\left|V-V_{j}\right| \forall j \wedge i \neq j\right\}
$$

Please refer to Figure 4(c). Having obtained preliminary nuclei segmentations using active contours, Voronoi partitioning of the image is accomplished using the nuclei centroid as generating points. Ideally, each individual nucleus lies within a corresponding Voronoi cell. In reality, this is not the case. A nucleus is anisotropic in shape, tends to overlap and stray across the Voronoi cell boundaries. Figure 4(d) provides one such example. Our methods alter the Voronoi tessellations to provide better nuclei separation by accounting for the overlap in various regions. The output from this stage is shown in Figure 4(e).

Note that the Voronoi representation of the tessellations given by Equation 5 is implicit and not parameterized. We instead use a framework that explicitly represents the Voronoi tessellations using parameters that in turn, can be suitably modified.

\section{Barycentric Representation of Tessellations - Step 2}

We review some basic aspects of barycentric coordinates with the help of Figure 5(a). Given the vertices $V=\left\{V_{1}, V_{2}, V_{3}\right\}$ of a Delaunay triangle $T$, any point $P(x, y)$ can be parameterized in terms of the barycentric coordinates $c=\left(c_{1}, c_{2}, c_{3}\right)$. The following equation when solved for $c$ gives us this representation:

$$
\left[\begin{array}{ccc}
V_{1 x} & V_{2 x} & V_{3 x} \\
V_{1 y} & V_{2 y} & V_{3 y} \\
1 & 1 & 1
\end{array}\right]\left[\begin{array}{l}
c_{1} \\
c_{2} \\
c_{3}
\end{array}\right]=\left[\begin{array}{l}
x \\
y \\
1
\end{array}\right]
$$

Note that the barycentric coordinates form a partition of unity, i.e, $c_{1}+c_{2}+c_{3}=1$. By definition, the vertices $V$ 


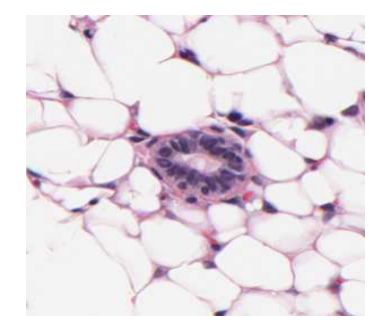

(a)

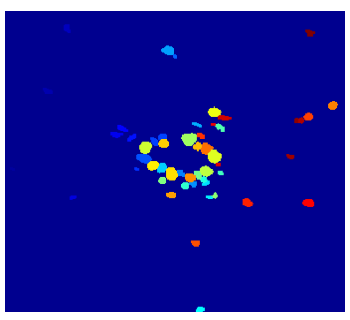

(b)

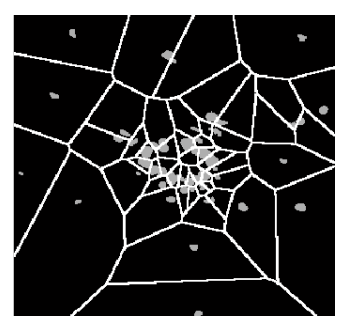

(c)

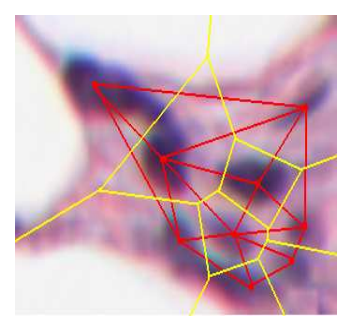

(d)

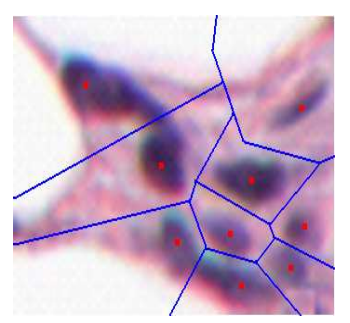

(e)

Fig. 4. Cellular segmentation pipeline (a) An example image of a duct cross-section formed by epithelial nuclei. (b) Segmentation of nuclei using level sets (c) Voronoi tessellation of the image plane.(d) Zoomed version of the Voronoi tessellations shown in yellow. (e) Optimal tessellation placement with nuclei well separated.

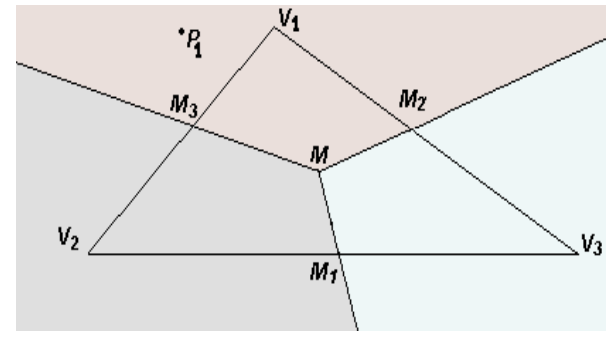

(a)

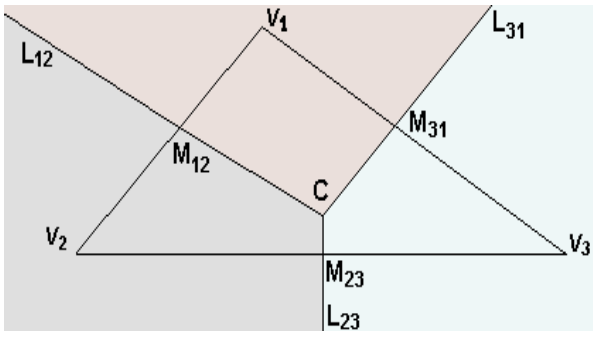

(b)

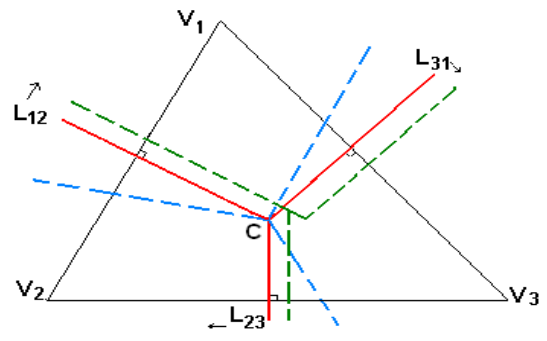

(c)

Fig. 5. (a) Barycentric coordinates based subdivision. (b) Tessellations unique to a Delaunay triangle $T$ are shown. The lines meet a at Voronoi vertex $C$ and are perpendicular bisectors of the triangle formed from the associated nuclei centroid.(c) Possible tessellation movements are shown with translated (green) and rotated (blue) edges starting from the Voronoi tessellation (red).

have barycentric coordinates of $(1,0,0),(0,1,0)$ and $(0,0,1)$ respectively. Using the barycentric variables $\left(c_{1}, c_{2}, c_{3}\right)$, the equation of a general line is given by:

$$
L^{T}: c_{i}-\lambda c_{j}=\delta \quad i, j \in\{1,2,3\}, i \neq j
$$

The parameters $(\lambda, \delta)$ represent the two degrees of freedom that are afforded in a equation describing a line. For example, by setting $(\lambda, \delta)$ as $(1,0)$ in the above equation results in three equations for the triangle medians which meet at the centroid $M$ (Figure 5a).

In Figure 5(b), we show a Voronoi cell vertex $C$ as local to a Delaunay triangle $T$ and common to three tessellation line segments $L_{12}, L_{23}$ and $L_{31}$ (solid lines). Using the barycentric representation, the equation of each tessellation line is:

$$
L_{i j}^{T}: c_{i}-\lambda_{i j}^{v} c_{j}=\delta_{i j}^{v} \quad \forall i, j \in\{1,2,3\}, i \neq j
$$

The Voronoi parameters $\left(\lambda_{i j}^{v}, \delta_{i j}^{v}\right)$ are uniquely solved by invoking the property that the tessellation line segments pass through mid-points $M_{i j}$ and $C$ (circumcenter of Delaunay triangle $T$ ). The work in [17] presents the solution in detail.

By changing the Voronoi parameters $\left(\lambda_{i j}^{v}, \delta_{i j}^{v}\right) \rightarrow\left(\lambda_{i j}, \delta_{i j}\right)$, the slope and intercept of each tessellation line $L_{i j}$ is adapted to achieve better nuclei separation. An example of the resulting line placements obtained by translating and rotating the Voronoi boundaries is shown by the dashed lines in Figure 5(c).

Changing the Voronoi tessellations in each Delaunay triangle and repeating this process over all triangles leads to better descriptions of the overall Voronoi tessellation (Figure 4(d) and 4(e)). Delaunay triangles have been marked in red in the figure. The new tessellations optimally house the nuclei and are more representative of the extent of nuclei infiltration.

\section{Estimating $(\lambda, \delta)$ - Step 3}

We automate the procedure of changing tessellation line parameters using a Bayesian probabilistic model of nuclei separation. This procedure is novel and allays the hesitancy in using global partitions like the Voronoi tessellations. For each line segment, we seek to estimate the maximum a posteriori parameters $\left(\lambda^{*}, \delta^{*}\right)$.

$$
\left(\lambda^{*}, \delta^{*}\right)=\arg \max _{\lambda, \delta} P\left(\lambda, \delta \mid S, I_{f}, f(I)\right)
$$

In this equation, $S$ is the initial coarse segmentation (Section III), $I_{f}$ is the nuclei foreground and $f(I)$ is as defined in Equation 3. Using Bayes rule,

$$
\begin{aligned}
& P\left(\lambda, \delta \mid S, I_{f}, f(I)\right)=\frac{P\left(S, I_{f}, f(I) \mid \lambda, \delta\right) P(\lambda, \delta)}{P\left(S, I_{f}, f(I)\right)} \\
= & \frac{P(S \mid \lambda, \delta) P\left(I_{f} \mid \lambda, \delta, S\right) P\left(f(I) \mid \lambda, \delta, S, I_{f}\right) P(\lambda, \delta)}{P\left(S, I_{f}, f(I)\right)}
\end{aligned}
$$

We discard the normalization term in the denominator as it does not influence $\lambda$ or $\delta$ and explain the remaining terms in turn.

Similarity Term: The first term $P(S \mid \lambda, \delta)$ denotes the posteriori conditional probability of achieving the coarse segmentation, $S$, given tessellation parameters $\lambda$ and $\delta$. Consider the illustration in Figure 6(a). We denote $(\widetilde{\lambda}, \widetilde{\delta})$ as the parameters 
of the least-squares fitted line separating the coarse segmentation boundary. Therefore, we write:

$$
P(S \mid \lambda, \delta)=P(\widetilde{\lambda}, \widetilde{\delta} \mid \lambda, \delta)
$$

Now, it is reasonable to assume that line parameters $(\lambda, \delta)$ when similar to $(\widetilde{\lambda}, \widetilde{\delta})$ results in a high probability of better segmentation as depicted in the figure. Again, dissimilar parameters means a displaced tessellation from the coarse segmentation case and therefore has a low probability. Hence, we model this term as a Gaussian density function over the squared difference in parameters:

$$
P(S \mid \lambda, \delta)=e^{-\left[(\widetilde{\lambda}-\lambda)^{2}+(\widetilde{\delta}-\delta)^{2}\right]}
$$

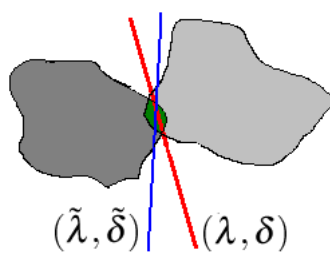

(a)

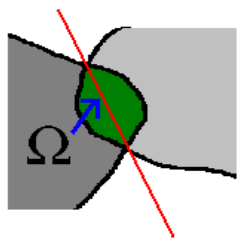

(b)
Fig. 6. (a) Illustration showing the optimal and segmented lines in the similarity term. (b) $\Omega$ shown as the intersection of nuclei foreground and the tessellation line

Overlap term: The neck region is defined as $\Omega=I_{f} \cap L(\lambda, \delta)$, i.e. the intersection of the nuclei foreground and the tessellation line. For an illustration, refer to Figure 6(b). Typically, the neck region is optimally narrow at the point of nuclei separation (see Figure 1(b) and Figure 3(c)). Hence, we model this term as:

$$
P\left(I_{f} \mid \lambda, \delta, S\right)=e^{-|\Omega|}
$$

Gradient Term: The third term $P\left(f(I) \mid \lambda, \delta, S, I_{f}\right)$ computes the conditional probability of the observed feature function $f(I)$ given the segmentation $(S)$ and optimal tessellation line $(\lambda, \delta)$. Typically, the function was designed in Equation 3 to have low values in $\Omega$. Hence, we write:

$$
P\left(f(I) \mid \lambda, \delta, S, I_{f}\right)=e^{-\int_{\Omega} f(I) d \Omega}
$$

Parameter priors: The last term is the Voronoi tessellation $\left(\lambda^{v}, \delta^{v}\right)$ applied as a prior. Recall that the tessellation is initialized as a Voronoi tessellation and hence in the absence of any information, we would like to maintain status quo.

$$
P(\lambda, \delta)=e^{-\left[\left(\lambda-\lambda^{v}\right)^{2}+\left(\delta-\delta^{v}\right)^{2}\right]}
$$

A gradient descent optimization is applied on Equation 9 to guide us to an optimal set of parameters $\left(\lambda^{*}, \delta^{*}\right)$. The barycentric parametrization serves to normalize all the tessellation lines to have the same optimal step lengths of the line parameters and convergence criterion. Normally, a Cartesian representation $(y=m x+c)$ of the lines would have different slope $(m)$ and intercept $(c)$ values. This causes the setting of initial parameters in the optimizer and in the MAP formulation to be line-specific, thereby making the problem intractable. The optimization procedure is repeated over all the tessellation line segments leading to new partitioning of the image space.
In Table II, we have listed parameter settings of our optimizer in our experiments. We have now obtained final nuclei segmentations on $2 D$ serial section images. The tessellations optimally house each nucleus and provide a better separation boundary in case of overlap. The $2 D$ nucleus segments form profiles of a $3 D$ nucleus whose shape parameters are estimated in the next section.

\section{Intermediate SLice Inter POLATION}

One of the major problems in reconstructing serial-sections has been different image plane and stacking resolutions. This problem is likely to be exacerbated in future, when in-plane resolutions improve exponentially while slicing thickness is likely to remain the same. A reduction of slice thickness is limited by the delicate nature of the soft tissues (in light microscopy) and the point-spread function of the optics (in confocal imaging).

For example, consider the situation in light microscopy. Typical inter-pixel spacing of about $0.46 \mu \mathrm{m}$ or even less is achieved normally. Meanwhile, the inter-slice spacing is limited to a range of 3-5 $\mu \mathrm{m}$. As a result, serial section stacks in histology present skewed voxel dimensions (1:1:10). In confocal microscopy, the image planes are resolved by the point-spread function (PSF) of the optics. The optics records light emanating from neighborhood of a physical voxel. Hence, image planes are once again constrained to be separated by a fixed depth albeit smaller than in light microscopy. However, the problems still persist.

Under-sampling artifacts arise when a nucleus is not sliced by a sufficient number of imaging planes. For example, the top row in Figure 7(a) displays a pair of consecutive sections with widely varying cellular arrangement patterns. Notice that the ductal cross-sections have changed significantly along with the arrangement of nuclei. The process of stacking the images will not allow the profiles of nuclei to correspond. Figure 1(c) and Figure 2(c) show other examples where stacking creates a linear interpolation of the nuclear material without any notion of its shape being considered. Patches with rapid intensity variation are then observed. However, $3 D$ cellular profiles are not visible since a nucleus is not sliced sufficiently fine (thin) by the sectioning process. Hence, there is no scope of reconstructing the intermediate variation between slices using information from two serial sections alone. We utilize a $3 D$ shape model in reconstructing the arrangement in intermediate slices as shown in the bottom row of Figure 7(b).

\section{A. 3D Cellular Shape Model}

A nucleus in a cell appears as a convex shape unless the cell is undergoing division. Depending on the state of a cell in the cell cycle, the shape of nucleus changes. Hence, an image plane is a snap-shot of cells at different phases in the cell cycle. These nuclei conform to a complex temporal shape model that is not known. We instead choose an average shape model to represent a general nucleus. To reconstruct $3 D$ nuclear shapes, we use a simple probabilistic model that treats a nucleus as an ellipsoid and its $2 D$ observations as the result of an orthographic projection [12]. 


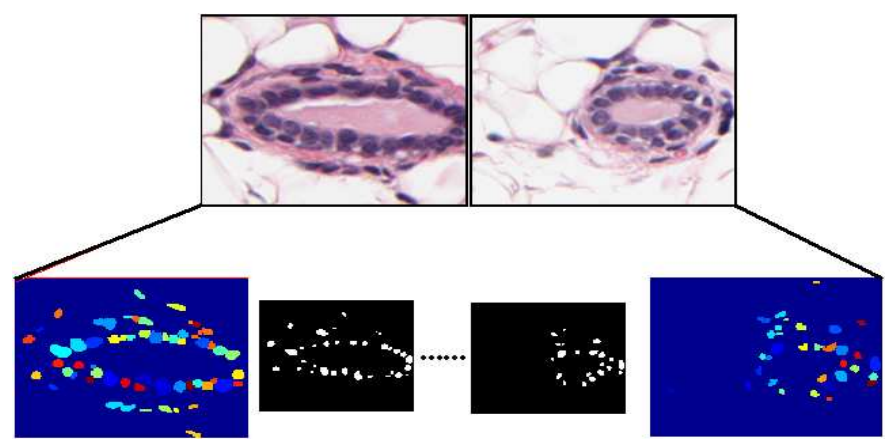

(a)

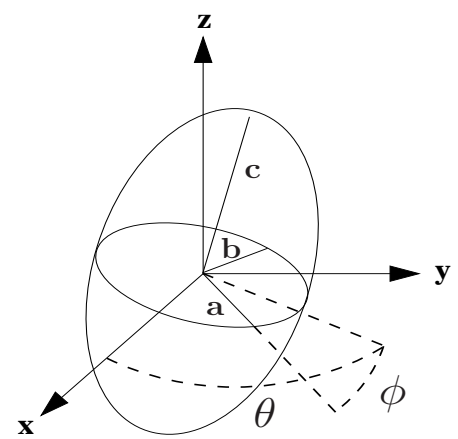

(b)

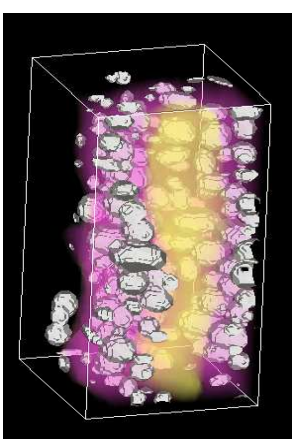

(c)

Fig. 7. (a) The top row shows two serial sections of the duct with significant variation in ductal shape and nuclei arrangement. The bottom row shows a interpolated sequence of processed nuclei profiles that gradually evolve from the start to the terminal image. (b) Ellipsoid nucleus model. (c) Isosurfaces of the nucleus-extracellular material are extracted after stacking. Notice the $3 D$ conic profiles of the nucleus are observable.

The model begins with the observation that nuclei observed in the images are an orthographic projection of their shapes onto the image plane. This is quite different from a crosssectional sample of a nucleus. Figure 7(b) shows the subject of our model, the ellipsoid with axis lengths $a, b, c$. The equation of a triaxial ellipsoid is given by:

$$
\frac{x^{2}}{a^{2}}+\frac{y^{2}}{b^{2}}+\frac{z^{2}}{c^{2}}=1
$$

For an ellipsoid with rotation and tilt angles $\theta, \phi$, the above equation is modified by applying a transformation to the coordinate axes. The transformation in the new reference frame is given by:

$$
\left[\begin{array}{l}
x \\
y \\
z
\end{array}\right]=\left[\begin{array}{ccc}
\sin \theta & \cos \theta \cos \phi & \cos \theta \sin \phi \\
\cos \theta & \sin \theta \cos \phi & -\sin \theta \sin \phi \\
0 & -\sin \phi & \cos \phi
\end{array}\right]\left[\begin{array}{c}
X \\
Y \\
Z
\end{array}\right]
$$

By substituting Equation 17 in Equation 16, we get the $3 D$ equation of an oriented ellipsoid. By setting $Z=0$, the $2 D$ orthographic projection of the ellipsoid on the $X-Y$ plane is obtained as:

$$
\begin{aligned}
\frac{(X \sin \theta+Y \cos \theta \cos \phi)^{2}}{a^{2}} & +\frac{(X \cos \theta+Y \sin \theta \cos \phi)^{2}}{b^{2}} \\
& +\frac{(-Y \sin \phi)^{2}}{c^{2}}=1
\end{aligned}
$$

It is easy to see that the two-dimensional ellipse parameters involves quadratic combinations of the ellipsoid parameters $(a, b, c, \theta, \phi)[11]$.

Given the nuclei segmentations, we first perform a least squares ellipse fit to each nucleus using [12] and resolve the axis lengths $(\alpha, \beta, \theta)$. Our task is to then estimate the five ellipsoid parameters given the three ellipse parameters. There are three non-linear, non-polynomial equations for estimating five parameters, which is clearly indeterminate.

We make simplifying assumptions to help our case. We assume that the projected ellipse has the same minor axis $(\beta=\mathrm{b})$ and $X-Y$ plane orientation $(\delta=\theta)$. Based on these assumption, the major axis is expressed as $\alpha=$ $\sqrt{a^{2} \cos ^{2}(\phi)+c^{2} \sin ^{2}(\phi)}$. Note that they share the same $(x, y)$ center location. To enforce a roughly circular cross-section, $a$ is drawn from a normal distribution with a mean value $b$. To retain the roughly prolate shape, $\phi$ is assigned from a normal distribution with mean $\pi / 2$. The values of $\alpha, a$ and $\theta$ uniquely determine $c$ from the relation $\alpha^{2}=a^{2} \cos ^{2}(\phi)+c^{2} \sin ^{2}(\phi)$.

\section{B. Shape Interpolation}

Given a serial-section image stack, cellular segmentation is conducted on the $2 D$ images using the methods outlined in Section III. Shape parameters $(a, b, c, \theta, \phi)$ for every nucleus in the segmentation is then estimated based on the ellipsoid projection model. Please refer to the bottom row in Figure 7(a). Between any two pair of serial sections, at least 9 intermediate slices are inserted. In other words, at least 9 extra slices of each $3 D$ nucleus are captured. Therefore, each nucleus, as abstracted by its ellipsoid parameters $(a, b, c, \theta, \phi)$ is sliced along the intermediate slice planes. Hence, the skew in voxel dimensions in the resulting stack of images is reduced to a isotropic ratio of $1: 1: 1$. Depending on the application, other cellular structures such as the duct tissue areas, vasculature etc. may also be interpolated onto the slides using appropriate models.

To reiterate, the end goal of the interpolation framework is to provide meaningful reconstructions of cell biology. While the $3 D$ information is captured by the $2 D$ images, simple stacking of the images does not resolve the issue. A cellular shape model allows us to extract the relevant information and therefore interpolate to provide $3 D$ descriptions. Please refer to Figure 7(c) wherein we show nuclei as salient isosurfaces embedded in the extracellular matrix. The nuclei surround a mammary duct that was earlier shown in Figure 1(c).

\section{RESUlTS}

In this section, we describe our results on three separate studies that invoke recurring themes of cell segmentation, $3 D$ reconstruction and visualization. Our methods are easily applied with changes in parameter settings to each of the data obtained from very different sources. In each study, we describe the data, the pertinent validation results, the computational performance of the reconstruction pipeline, and the resulting visualization. 

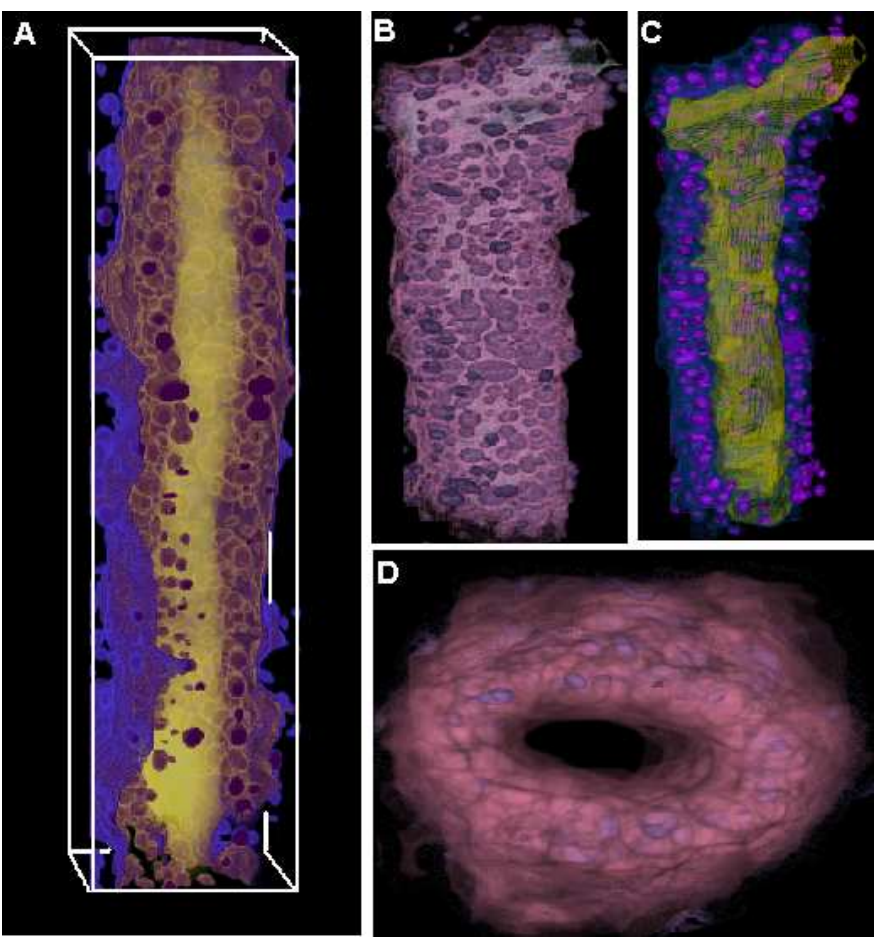

Fig. 8. Enhanced visualization of duct sequences in comparison to Figure 1c. (A) Segmented volume consisting of nuclei, extracellular matrix, lumen and the duct. (B) True color rendering showing the epithelial nuclei surrounding the duct. (C) Epithelial nuclei lying around a branching duct. A cut-away section reveals the underlying lumen. (D) Top-view of the duct with the seethrough hole.

\section{A. Mouse Model Phenotyping Studies}

In this work, our collaborator (co-author: Leone, The Ohio State University) is interested in understanding the role of the PTEN tumor suppressor gene in the regulation of normal cellular processes. Phenotyping experiments on mouse mammary models have revealed that suppression of PTEN gene leads to abnormal cellular transformations. Our collaborator seeks to understand the orchestrated sequence of events leading to tumor initiation and progression at a cellular as well as a molecular scale. In this context, a full fledged spatial reconstruction of the ductal tissue can significantly reduce the time required to generate new hypotheses regarding the cellular mechanisms. A pair of wild-type mouse mammary glands were harvested and prepared using a standard histological protocol. They were fixed in formalin, embedded in paraffin and sectioned at $5 \mu \mathrm{m}$ thickness using a microtome to yield about 1000 sections per dataset. Serial sections were mounted on glass slides and scanned at 200x magnification (0.46 $\mu \mathrm{m}$ inter-pixel spacing) using a light microscope. The image dimensions on average were $(15 \mathrm{~K} \times 15 \mathrm{~K})$ in $\mathrm{RGB}$ format.

Mammary ductal tissue is composed of concentric layers of epithelial in a matrix of extracellular material. The ducts are identified on each section as a distinct conic or quadric projection of a complex tortuous $3 D$ object (Figure 1(a)). We used the $\mathrm{N}$-point correlation functions as feature vectors to classify ductal tissue regions [21]. These functions measure the packing of the cells and are shown to provide robust classification. Rigid registration was accomplished using a global alignment procedure [18] followed by a refined local matching of individual ductal areas [6]. $3 D$ profiles of the ducts were extracted from the large dataset to yield smaller volumes of $300 \times 300 \times 34$ per duct for processing. We then apply the tools described in this work to the problem of providing duct reconstructions at a cellular scale.

Individual nuclei were identified in 2D images and overlapping nuclei clusters were split consistently using the two successive segmentation stages based on active geodesic contours and tessellations. We then performed interpolation of intermediate slice locations as described in Section $\mathrm{V}$ to reconstruct $3 D$ ductal volumes for visualization. A total of 18 contiguous duct sequences were reconstructed using the above automated procedure.

Figure 8 provides examples of 4 such sequences. Panel A is a volume rendering of a segmented duct using a cutting plane parallel to the duct axis. The elliptic nuclei profiles are visible in the ductal wall. Panel B is the true color rendering with interpolated textures of the ductal tissue. It is easy to notice the lack of any cellular descriptions in the rendering. Panel $\mathrm{C}$ shows yet another branching duct with nuclei and lumen alone. A hemi-cylindrical cut away section was used to reveal the underlying lumen. Panel D is a top-view of the duct revealing a constellation of cells along the duct akin to a brick chimney wall.

\section{B. Cell Colonies in Clonogenic Assays}

It is quite well established that tumors have a clonal origin - all of the affected and transformed cells can be traced to a small set of mutated precursor cells that lose the ability to die (apoptosis - programmed cell death) and hence continue to proliferate [10]. In in-vitro cell cultures, it is observed that mutated cells tend to form colonies due to rapid proliferation. Given their need to develop novel therapies for cancer, our collaborators (Jian Chen and Weiming Xia, Harvard Medical School) are interested in determining the effect of certain chemicals in preventing or reducing the colony growth in mutated cells. Hence, normal cells in culture were exposed to radiation to undergo mutation and later treated with an appropriate chemical. The wells were then observed using phase-contrast microscopy at various fixed time-points. The goal of image analysis is to detect cells, determine their membership to different cell colonies and track their growth across the frames. We do not report on any tracking results here.

The images were acquired by General Electric IN CELL Analyzer 1000 microscope with a $10 X$ objective. The images were collected over cultured cells that were placed in 96-well plates. Each dataset consists of three (3) time frames each of which having image dimensions of approximately $1000 \times$ 1000 (on average). We applied our $2 D$ cell segmentation methods to 10 images. This data is employed for the sole purpose of testing the segmentation methods. Additionally, the nuclei in this data are not typically spherical like other data. However, it should be noted that our methods can be deployed quite easily. Figure 9 provides examples of the segmentation performance on a single dataset. Figure 9(a-c) 


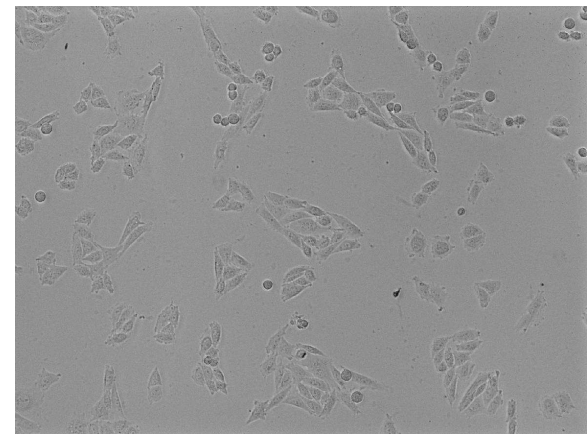

(a)

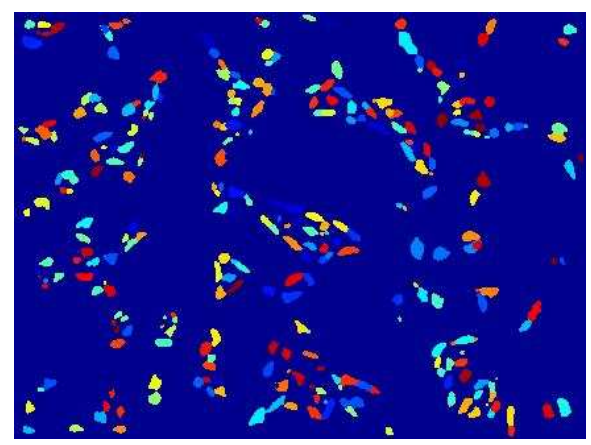

(d)

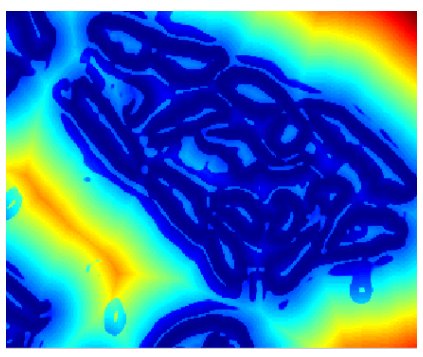

(g)

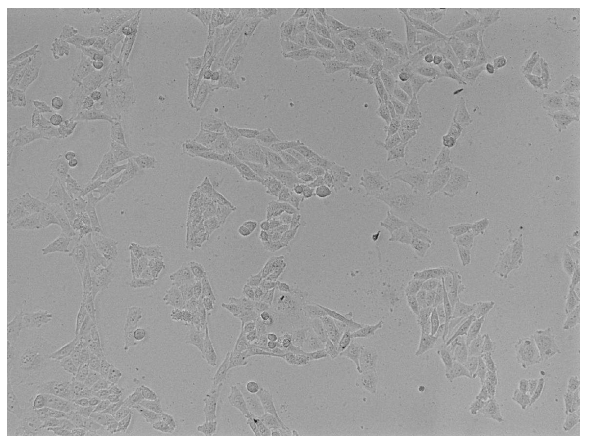

(b)

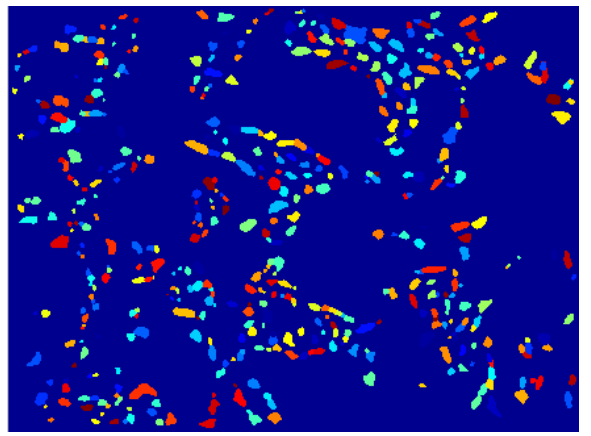

(e)

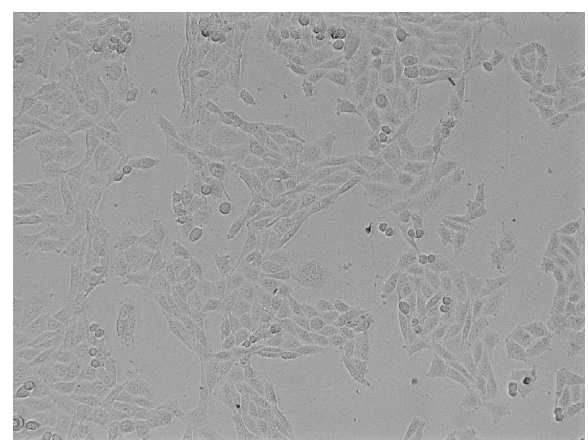

(c)

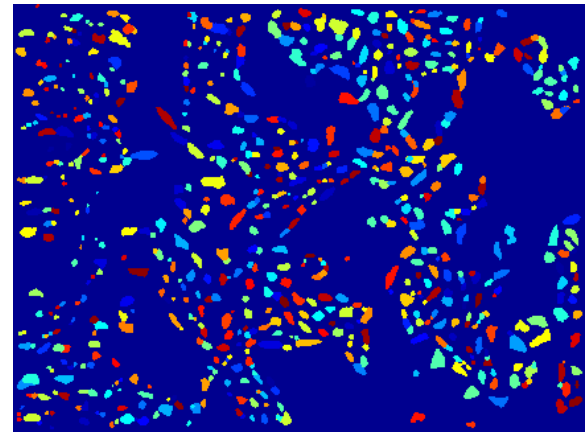

(f)

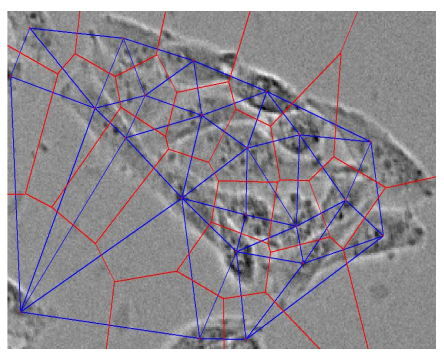

(h)

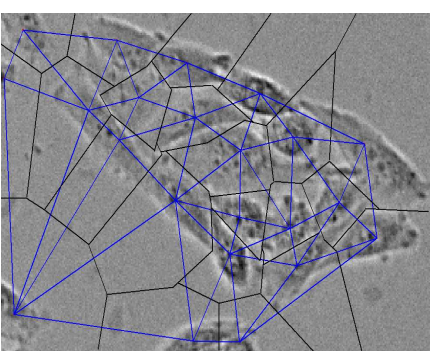

(i)

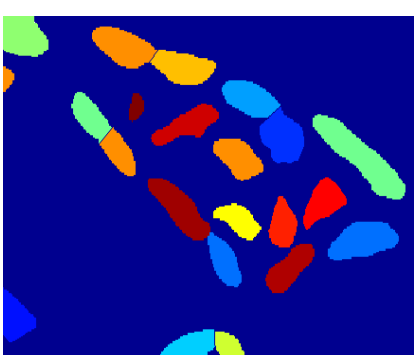

(j)

Fig. 9. Image frames of cells at (a) $0^{\text {th }}$ hour, (b) $12^{\text {th }}$ hour and (c) $24^{\text {th }}$ hour. (d,e,f) Cell segmentations of images at respective time steps. (g) Feature function $f(I)$ of the zoomed portion of cells from Figure 2. It is easy to notice the role of gradients and the distance field in creating minima at regions of overlap. (h) Voronoi partitioning overlaid on the original figure. (i) Modified tessellations. (j) Final cell segmentations obtained after splitting overlapping cells.

are the original raw acquisitions from the microscope while (d-f) show the segmentations of each cell. Note that each cell is represented by a unique number in the range [0-255]. Since the number of cells are more than 255 , some of the colors are repeated in the images although they correspond to different cells. It is easy to notice that in the first frame, cells cluster together in small colonies. In Figure 9(d) and Figure 9(e), these colonies multiply and consist of exponentially increasing number of cells. In Figure 9 ( $\mathrm{g}-\mathrm{j})$, we provide a slide-show of the intermediate steps in the cell segmentation pipeline using a zoomed version of the data. Figure $9(\mathrm{~g})$ shows the feature function $f$ on the set of colony cells. The tessellations are then used to refine the segmentation and are overlaid on the original image for verification $(h, i)$. Figure $9(j)$ depicts the result of the application of the tessellation for getting the final segmentation. The later stages of our pipeline including interpolation and $3 D$ reconstruction were not applicable in this study.

\section{Zebra Fish Phenotyping Studies}

Our collaborators at The Center of Excellence in Genomic Science at California Institute of Technology (Caltech) (Alexandre Gouaillard, Titus Brown, Marianne BronnerFraser, Scott E. Fraser and Sean Megason) have initiated the Digital Fish Project. Their goal is to use in toto imaging of developing transgenic zebrafish embryos to acquire digital, quantitative, cell-based, molecular data suitable for modeling the biological circuits that turn an egg into an embryo. In toto imaging uses confocal microscopy to capture the entire volume of organs and eventually whole embryos at cellular resolution every few minutes in living specimens throughout their development. $4 D$ image sets $(x-y-z-t)$ with dimensions $1024 \times 1024 \times 80 \times 60$ are generated. The plan is to use in toto imaging to digitize the complete expression and subcellular localization patterns of thousands of proteins throughout zebrafish embryogenesis. We provide $3 D$ results from applying our methods at select time-points chosen at 0 


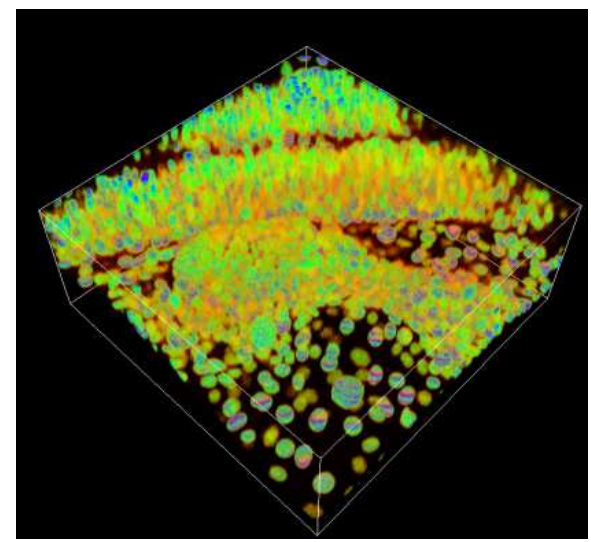

(a)

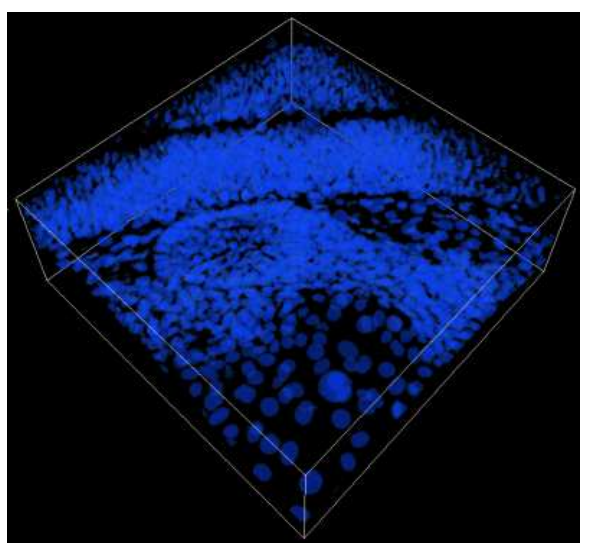

(b)

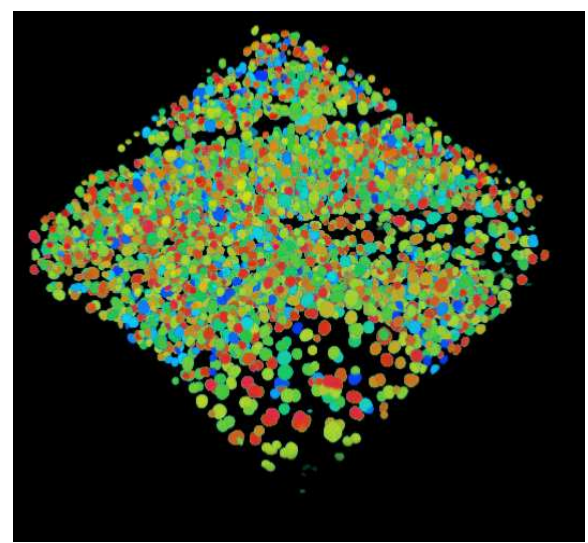

(c)

Fig. 10. $3 D$ cellular reconstructions from confocal images of the zebrafish embryo at time-point 60. (a) Raw data renderings, (b) foreground extractions, and (c) cell segmentations.

(beginning) and 60 (ending) respectively.

Figure $10(\mathrm{a}-\mathrm{c})$ depict the rendering of original raw data, foreground and cell segmentation respectively. Note that once again the repetition in colors is owing to the large number of cells.

\section{Validation}

We evaluate the utility of the segmentation framework in effectively segmenting nuclei that appear as clusters. Validation studies are performed with manually marked nuclei areas. It is very difficult to manually identify all the valid nuclei in a dataset. Typically, the number of nuclei can be in the order of several thousands. Therefore, we sample ten regions of dimensions $100 \times 100$ in an unbiased fashion from each dataset. All the nuclei in these windowed regions are marked out to obtain classified masks that serve as groundtruth. The matched pair of nuclei can be separated and counted in three categories: (i) a counts the number of true-positive results. (ii) $b$ counts the number of false-negative results. (iii) $c$ counts the number of false-positive results. Note that falsenegative results cannot be determined since we have no $a$ priori information on where a nuclei should not have existed. Thus, one can define the sensitivity and positive predictive value (PPV) of our framework as follows:

$$
\text { sensitivity }=\frac{a}{a+b} \quad \mathrm{PPV}=\frac{a}{a+c}
$$

\begin{tabular}{|c|c|c|}
\hline Dataset & Dimension & Cells \\
\hline Clonal 1 & $1024 \times 1024$ & 378 \\
\hline Clonal 2 & $1024 \times 1024$ & 610 \\
\hline Clonal 3 & $1024 \times 1024$ & 794 \\
\hline Ducts & $300 \times 300 \times 34$ & 1710 \\
\hline ZebraFish & $1024 \times 1024 \times 80$ & 4130 \\
\hline
\end{tabular}

TABLE I

TEST DATASETS CHOSEN FOR VALIDATION STUDIES

Table I lists our test datasets with dimensions and the total number of nuclei actually segmented throughout the dataset.
The test datasets include a clonal assay dataset consisting of three (3) temporal frames, a mammary duct dataset containing thirty four (34) slices and a zebrafish confocal dataset having eighty (80) slices.

\begin{tabular}{|c|c|c|c|}
\hline Dataset & Cells & Sens. & PPV \\
\hline Clonal 1 & 74 & 0.9324 & 0.9452 \\
\hline Clonal 2 & 117 & 0.8547 & 0.9259 \\
\hline Clonal 3 & 174 & 0.8996 & 0.9455 \\
\hline Ducts & 217 & 0.9724 & 1 \\
\hline ZebraFish & 252 & 0.8968 & 0.9826 \\
\hline
\end{tabular}

TABLE II

SENSITIVITY AND PPV VALUES FOR CELL SEGMENTATION

From Table II, we observe that our methods yield an average value $94 \%$ and $96 \%$ for sensitivity and PPV respectively. Sensitivity measures the effectiveness of the algorithm in detecting the true-positives. A high value indicates that it is easy for the algorithm to rule out a spurious match with a high probability. On the other hand, the positive predictive value estimates the proportion of segmented nuclei which are bona fide ones. The false-positives are mostly due to undersegmentation - the true cell region is found to be grouped in a single larger segmented region. Similarly, the true-negatives are due to over-segmentation - the true cell region is found to have multiple segmentations.

Validation studies were also performed using the same protocol as described in [4]. In this case, a histogram of signed distances of the nucleus contour with respect to the groundtruth contour is computed for all the true-positive results generated from the previous study. In Figure 11, we plot the distribution of distance of the segmentation boundary pixels from the manual boundary. We observe that in the case of the mammary ducts (red markers), ninety percent $(90 \%)$ of the boundary pixels in the automatic segmentation are within 3 pixels in distance from the corresponding manual boundary. Similarly, seventy-five percent $(75 \%)$ of the boundary pixels are within 2 pixel distances. The figure also shows that similar trends were observed in the case of the confocal images of the zebra-fish (blue markers). 


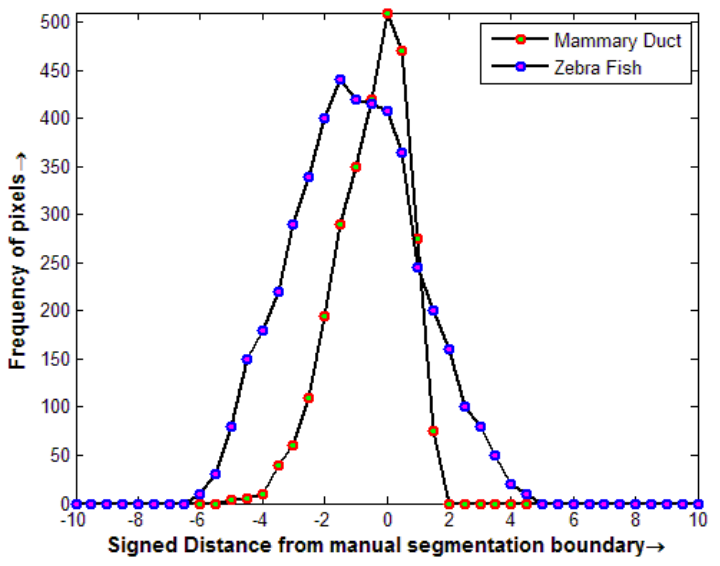

(a)

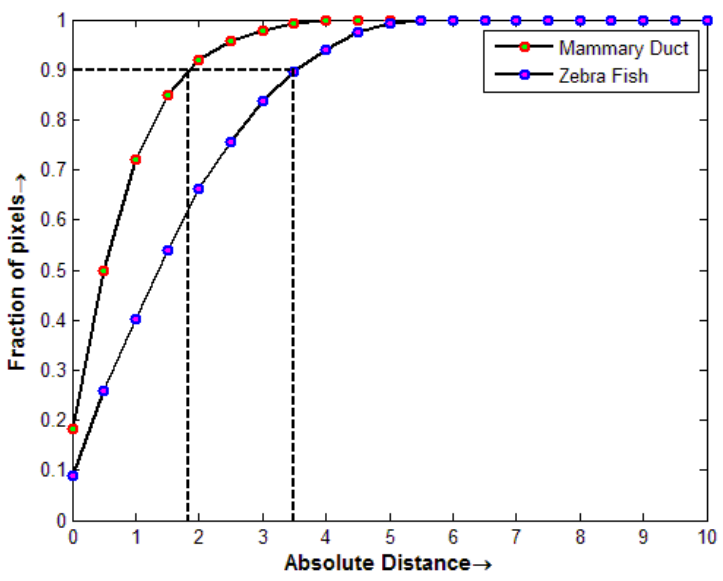

(b)

Fig. 11. (a) Combined histogram for the signed distances found in the mammary and confocal datasets and (b) cumulative distribution of absolute distances from automatic segmentation to manual segmentation.

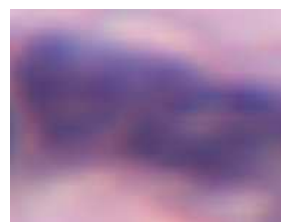

(a)

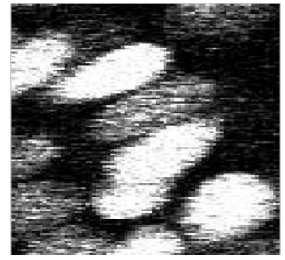

(d)

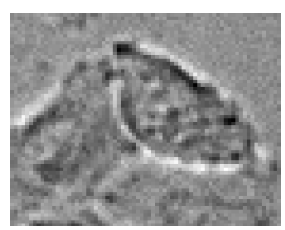

(g)

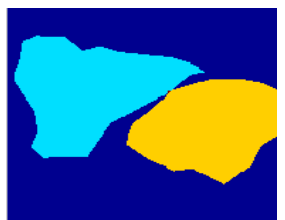

(b)

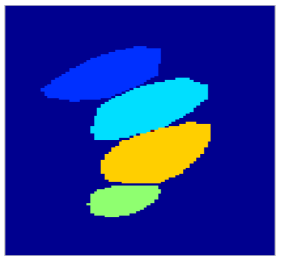

(e)

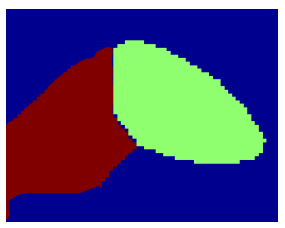

(h)

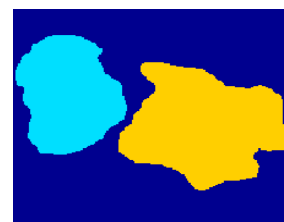

(c)

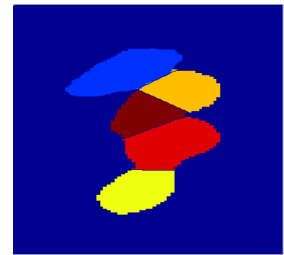

(f)

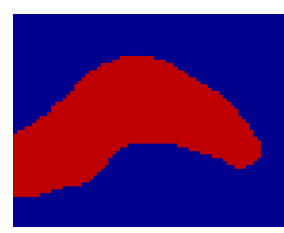

(i)
Fig. 12. Instances of outliers in the segmentation process with the top row showing a mammary duct sample with an inconsistent split, the middle row showing a zebrafish image with over-segmentation and the bottom row from a clonal colony with under-segmentation. (a,d,g) Original images, (b,e,h) manual masks and $(\mathrm{c}, \mathrm{f}, \mathrm{i})$ segmentations.

We also examined the segmentation outliers from the test data sets as shown in Figure 12. We present three scenarios that generate the outliers. In the top row, the automatic method has "chosen" to split a nuclei cluster differently (Figure 12(c)) from what an expert human would do (Figure 12(b)). On close examination (Figure 12(a)), we found very weak gradient and neck cues. This was a difficult case for performing manual segmentation as well. The middle row (Figure 12(d-f)) is marked by over-segmentation. Note that the active contour parameter settings were uniformly applied for all nuclei. However, certain nuclei present variations in shape, color luminance and gradients. This leads to multiple segmentations of the same nucleus. In the bottom row (Figure 12(g-i)), the poor gradients and large overlaps cause an under-segmentation and leakage of the level-set into neighboring nuclei.

\section{E. Implementation}

We implemented our framework using the National Library of Medicine's (NIH/NLM) Insight Segmentation and Registration Toolkit (ITK) and the Visualization Toolkit (VTK) from Kitware Inc. The classified volumetric datasets are loaded into Kitware's VolView volume visualization software to render the surface appropriately. All our tasks were conducted on a $2.5 \mathrm{GHz}$ Pentium machines running Linux with $1 \mathrm{~GB}$ main memory.

\begin{tabular}{|c|c|c|c|}
\hline Dataset & Active Contours & Tesl. & Interp. \\
\hline Clonal 1 & 201 & 131 & - \\
\hline Clonal 2 & 363 & 238 & - \\
\hline Clonal 3 & 442 & 376 & - \\
\hline Duct & 1183 & 126 & 145 \\
\hline ZebraFish & 2473 & 743 & 176 \\
\hline
\end{tabular}

TABLE III

COMPUTATION PERFORMANCE FOR THE TEST DATASETS IN SECONDS

The total running time comprises of the time expended in three stages, $T=T_{\text {LevelSet }}+T_{\text {Tess }}+T_{\text {Interp }}$. The active contour segmentation was observed to be the most expensive phase of the pipeline. In our implementation, the active contours routine is realized as a function call with the nucleus initialization and the speed image being provided as inputs. Since the segmentation is local to the nucleus initialization, we operate only on a small windowed neighborhood. The net result effectively reduces the time consumed significantly. Using our optimized implementation, we process a duct sequence in under 1500 seconds (25 minutes). The active contour segmentation expends 1200 seconds while tessellations and interpolation expend less than 150 seconds each. Table III lists our running times recorded on the validated test datasets in seconds. 


\begin{tabular}{|l|c|c|c|}
\hline Parameter Description & Clonal & Duct & Confocal \\
\hline$T$ - Foreground threshold (eq. 2) & 70 & 150 & 140 \\
$\sigma$ - Gaussian kernel (sec. III) & 1.0 & 1.0 & 1.0 \\
$\alpha$ - Attraction term (eq. 1) & 1 & 1 & 1 \\
$\beta$ - Advection term (eq. 1) & 3 & 3 & 3 \\
$\gamma$ - Diffusion term (eq. 1) & 3 & 3 & 3 \\
$\delta$ - Model update (eq. 1) & 2 & 2 & 2 \\
$N_{l s}$ - Max. level-set iters. (sec. III) & 400 & 400 & 400 \\
$R_{l s}$ - Max. rms change (sec. III) & 0.03 & 0.03 & 0.03 \\
$\eta$ - User-defined weights (eq. 3) & 0.8 & 1 & 0.5 \\
- Min. step length (sec. IV-C) & 0.01 & 0.01 & 0.01 \\
- Max. step length (sec. IV-C) & 0.25 & 0.25 & 0.25 \\
- Max. optimizer iters. (sec. IV-C) & 10 & 10 & 10 \\
\hline
\end{tabular}

TABLE IV

VALUES USED IN THE ITK IMPLEMENTATION.

Table IV lists the typical parameter settings that were used in our experiments. The parameters $T$ and $\eta$ are the two most critical settings that need to be explored for a given dataset. In our datasets, the staining protocol (nuclei-specific) is designed to provide a good contrast of the nuclei from the rest of the image. Figure 13 shows the histograms of a duct cross-sectioal image and a confocal zebrafish image respectively. There is a distinct valley region (indicated by arrows) that separates the nuclei from the rest of the image. Hence, the threshold parameter $T$ does enjoy a good dynamic range of operation. The value of this parameter is dictated by the instrument or the modality.

Our implementation explored a range of $\eta$ values in Equation 1 depending on the dataset. For example, in the ductal dataset, $\eta$ was set to 1 thereby assigning equal weights to the shape cues as well as the gradients. Figure 14 reports the sensitivity and PPV values as a function of different values of $\eta$ for the mammary duct dataset. We observe similar validation results for a broad range $([0.5,1.5])$ in the settings of $\eta$. Higher values tend to assign more importance to shape cues leading to fragmentation of nuclei (over-segmentation) and hence lower the value of PPV. Lower values tend to retain nuclei clusters without splitting them (under-segmentation) thereby lowering the sensitivity value. Hence, a judicious choice is recommended.

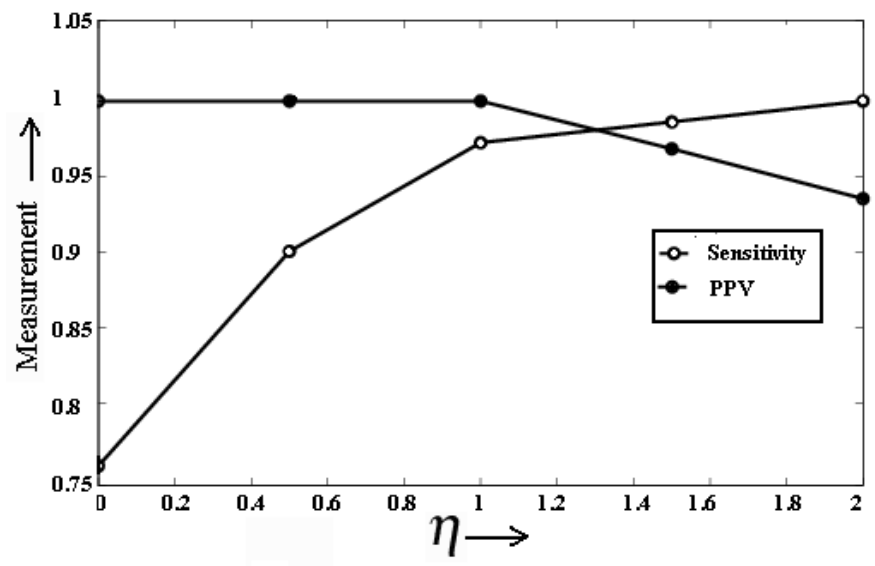

Fig. 14. A plot of the sensitivity and PPV values upon changing $\eta$ values for the mammary duct dataset.

\section{Summary AND Future Work}

In this paper, we addressed challenges in the $3 D$ reconstruction of cellular structures in microscopy datasets, namely: providing scalable cell segmentations and correcting axial under-sampling artifacts. We realized a segmentation approach using active contour models that is refined by means of image tessellations. Overlapping nuclei clusters are consistently cleaved by the tessellations. Insufficient slicing resolution is resolved by interpolating intermediate slices with a $3 D$ nucleus shape model. Our methods have been applied across modalities to data generated from light, confocal and phasecontrast microscopy. The algorithms were validated using manually verified ground-truth. In future, we wish to generate a realistic reconstructed model of the tumor microenvironment and to provide the biomedical research community with a phenotyping tool based on the imaging framework developed here. Where possible we will explore the development of complete $3 D$ methods; this is can be achieved for confocal microscopy of very small organ structures.

\section{ACKNOWLEDGEMENTS}

We gratefully thank the researchers providing images for this study: Jian Chen, Weiming Xia (Center for Neurologic Disease, Brigham and Women's Hospital, Harvard Medical School, Clonogenic assay dataset from phase-contrast microscopy), Alexandre Gouaillard, Titus Brown, Marianne Bronner-Fraser, Scott E. Fraser and Sean Megason (The Center of Excellence in Genomic Science, Caltech, Zebra fish confocal dataset), Stephen Aylward (Kitware Inc., USA) and Steve Pieper (Isomics Inc., USA)).

\section{REFERENCES}

[1] U. Adiga and B. Chaudhuri. An efficient method based on watershed and rule-based merging for segmentation of 3-d histo-pathological images. Pattern Recognition, 34(7):1449-1458, 2001.

[2] A. Almsherqi, S. Kohlwein, and Y. Deng. Cubic membranes: a legend beyond the flatland of cell membrane organization. Cell Biology, 173:839-844, 2006

[3] U. Braumann, J. Kuska, J. Einenkel, L. Horn, M. Löffler, and M. Höckel. Three-dimensional reconstruction and quantification of cervical carcinoma invasion fronts from histological serial sections. IEEE Transactions in Medical Imaging, 24(10):5-7, 2005.

[4] A. Carpenter, T. Jones, M. Lamprecht, C. Clarke T. Kang, O. Friman D. Guertin J. Chang, R. Lindquist J. Moffat, P. Golland, and D. Sabatini. Cellprofiler: image analysis software for identifying and quantifying cell phenotypes. Cell Biology, 7(R100), 2006.

[5] K. Conor, H. Voorheis, and C. Ó Sullivan. 3D visualisation of confocal fluorescence microscopy data. In Proceedings of the Fifth Irish Workshop on Computer Graphics, pages 49-54, 2004.

[6] L. Cooper, K. Huang, A. Sharma, K. Mosaliganti, and T. Pan. Registration vs. reconstruction: Building 3-d models from 2-d microscopy images. In Proceedings of Workshop on Multiscale Biological Imaging, Data Mining and Informatics, pages 57-58, 2006.

[7] Q. Du and X. Wang. Centroidal voronoi tessellation based algorithms for vector field segmentation and visualization. In Proceedings of IEEE Visualization, pages 43-50, 2004.

[8] R. Fernandez-Gonzalez, T. Deschamps, A. Idica, R. Malladi, and C. de Solorzano. Automatic segmentation of histological structures in mammary gland tissue sections. Biomedical Optics, 9(3):444-453, 2004.

[9] J. Fiala and K. Harris. Computer-based alignment and reconstruction of serial sections. Microscopy and Analysis, 52:5-7, 2002.

[10] P. Fialkow. Clonal origin of human tumors. Annual Review of Medicine, 30:135-143, 1979. 


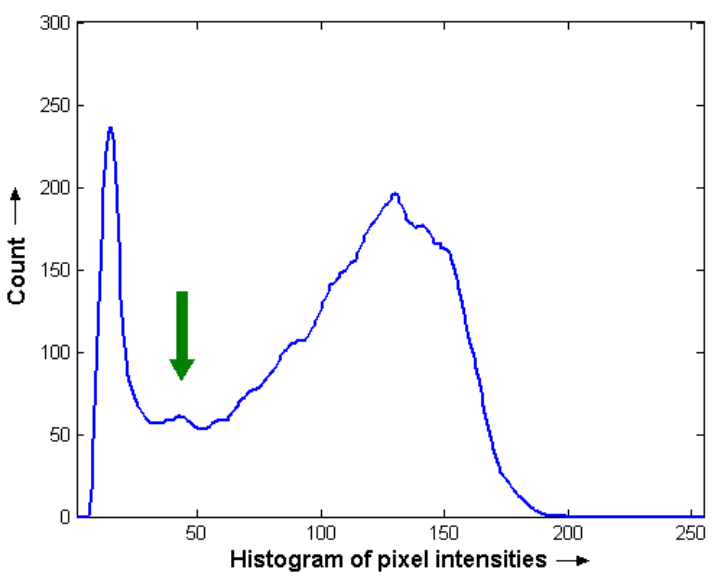

(a)

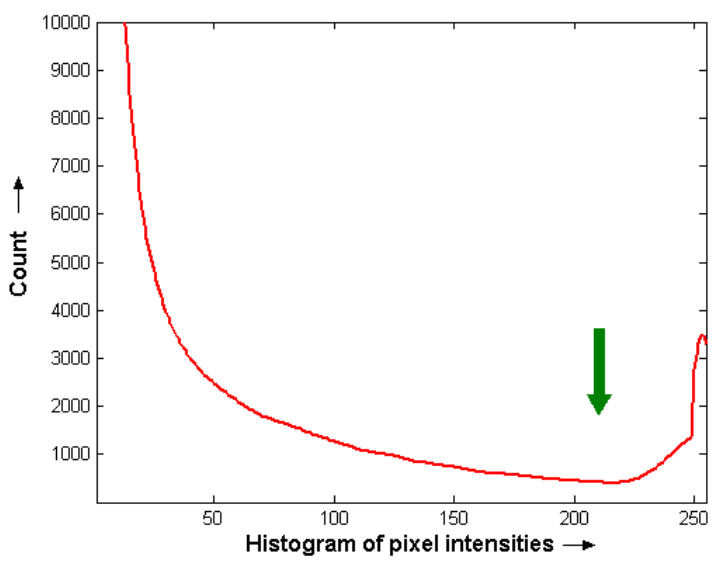

(b)

Fig. 13. Image histograms of (a) sample mammary gland slice, and (b) confocal image of zebra fish.

[11] D. Gendzwill and M. Stauffer. Analysis of Triaxial Ellipsoids: Their Shapes, Plane Sections, and Plane Projections. Springer Netherlands, 1981.

[12] R. Halir and J. Flusser. Numerically stable direct least squares fitting of ellipses. In Proceedings of the International Conference in Central Europe on Computer Graphics, Visualization and Interactive Digital Media, pages 125-132, 1998.

[13] A. Kaufman, R. Yagel, R. Bakalash, and I. Spector. Volume visualization in cell biology. In Proceedings of IEEE Visualization, pages 160-167, 1990.

[14] K. Kurose, K. Gilley, S. Matsumoto, P. Watson, X. Zhou, and C. Eng. Frequent somatic mutations in PTEN and tp53 are mutually exclusive in the stroma of breast carcinomas. Nature Genetics, 32(3):355-357, 2002.

[15] M. Leventon, W. Grimson, and O. Faugeras. Statistical shape influences in geodesic active contours. In Proceedings of the IEEE Conference on Computer Vision and Pattern Recognition, pages 316-232, 2000.

[16] N. Malpica, C. Solorzano, J. Vaquero, A. Santos, I. Vallcorba, J. GarciaSagredo, and F. Poze. Applying watershed algorithms to the segmentation of clustered nuclei. Journal of Cytometry, 28:289-297, 1997.

[17] K. Mosaliganti, R. Machiraju, J. Heverhagen, J. Saltz, and M. Knopp. Exploratory segmentation using geometric tessellations. In Proceedings of Tenth International Fall Workshop on Vision, Modeling and Visualization, pages 1-8, 2005.

[18] K. Mosaliganti, T. Pan, R. Sharp, R. Ridgway, A. Gulacy, S. Iyengar, P. Wenzel, A. deBruin, R. Machiraju, K. Huang, G. Leone, and J. Saltz. Registration and 3D visualization of large microscopy images. In SPIE Conference on Medical Imaging, pages 923-934, 2006.

[19] T. Ohtake, I. Kimijima, T. Fukushima, H. Yasuda, M. Sekikawa, S. Takenoshita, and R. Abe. Computer-assisted complete threedimensional reconstruction of the mammary ductal/lobular systems. Cancer, 91(12):2263-2314, 2001.

[20] S. Raman, B. Parvin, C. Maxwell, and M. Barcellos-Hoff. 3d segmentation of mammospheres for localization studies. In International Symposium on Visual Computing, pages 427-436, 2005.

[21] R. Ridgway, O. Irfanoglu, R. Machiraju, and K. Huang. Image segmentation with tensor-based classification of N-point correlation functions. In MICCAI Workshop on Medical Image Analysis with Applications in Biology, 2006.

[22] G. Sakas, M. Vicker, and P. Plath. Visualization of laser confocal microscopy datasets. In Proceedings of IEEE Visualization, pages 375379, 1996.

[23] R. Sharp, R. Ridgway, K. Mosaliganti, P. Wenzel, T. Pan, A. Bruin, R. Machiraju, K. Huang, G. Leone, and J. Saltz. Volume rendering phenotype differences in mouse placenta microscopy data. Special Issue on Anatomic Rendering and Visualization, Computing in Science and Engineering, 9(1):38-47, 2007.

[24] C. Tang and B. Ewert. Automatic tracking of neural stem cells. In Workshop Proceedings of WDIC 2005, pages 61-66.

[25] M. Tscherepanow, F. Zöllner, and F. Kummert. Automatic segmentation of unstained living cells in bright-field microscope images. In Workshop on Mass-Data Analysis of Images and Signals in Medicine, Biotechnology and Chemistry MDA, pages 86-95, 2006.

[26] Q. Wang, Y. Sun, B. Rajwa, and J. Robinson. Interactive volume visualization of cellular structures. In Proceedings of the SPIE, Volume 6065, pages 424-435, 2006.

[27] Q. Wang, Y. Sun, and J. Robinson. GPU-based visualization techniques for 3D microscopic imaging data. In Proceedings of the SPIE, Volume 6498, page 6498H, 2007. 